\title{
Intervención educativa en orientación alimentaria en jóvenes de 18 a 29 años en la facultad de medicina de la Universidad Autónoma de Nuevo León
}

Educational intervention in dietary guidance in subjects 18 to 29 years at the faculty of medicine of the Universidad Autónoma de Nuevo León

Intervenção educativa na orientação food 18 a 29 anos na faculdade de medicina da Universidade Autônoma de Nuevo León

Luis Castillo Reyna1 ^, Graciela Martínez Tamez', María Rodríguez', Adriana López Cárdenas', Mónica Llaca Hernández', Angel Castillo Reyna', David Castañeda Rodríguez'

Fecha correspondencia:

Recibido: mayo 4 de 2016.

Revisado: junio 1 de 2016.

Aceptado: junio 20 de 2016.

Forma de citar:

Castillo L, Martínez G, Rodríguez

M, López A, Llaca M, Castillo

A, Castañeda D. Rev CES Salud

Pública. 2016; 7 (2)

Open access

(C) Copyright

Licencia creative commons

Ética de publicaciones

Revisión por pares

Gestión por Open Journal System

ISSN: 2145-9932

Sobre los autores:

1. Facultad de Medicina de la

Universidad Autónoma de Nuevo León,

Depto. de Medicina Preventiva y Salud

Pública.

Comparte

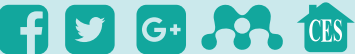

\section{Resumen}

Objetivos: correlacionar si la educación en orientación alimentaria y actividad física es una medida eficaz para la reducción de peso en estudiantes de la facultad de Medicina de la UANL de 18 a 29 años que cursan el segundo año de la carrera de médico cirujano y partero. Material y métodos: estudio de cohorte; se realizo con la totalidad de los estudiantes de la UANL que cursaron el segundo año de la carrera de médico cirujano y partero. Se formaron 2 grupos: grupo A en el cual se llevó a cabo una intervención educativa y grupo B de control; se volvieron a evaluar ambos grupos después de 6 meses para evaluar su progreso y por finalización de semestre a los 6 meses. Resultados: un total de 776 (n) se dividieron en grupo A (389) y grupo B (387). En el grupo A el peso basal $73.96 \mathrm{Kg} \mathrm{DE}$. \pm 17.28 y su IMC 25.73 DE. \pm 4.89. Posterior a la intervención se encontró que el peso fue de 71.59 D.E. \pm 16.4 , disminución de $2.37 \mathrm{Kg} \mathrm{DE} \pm 1.73 \mathrm{Kg}$. Del grupo B peso inicial 63.66 DE \pm 12.98 , e IMC 22.81 DE \pm 3.55 . En la segunda evaluación se encontró un peso promedio de 64.24 DE \pm 12.81 , hubo aumento de peso de $0.58 \mathrm{Kg} \mathrm{DE} \pm 0.64$. Hubo perdida de peso en grupo A en comparación a grupo $B(p=0.000021)$. Conclusiones: En el grupo A hubo perdida significativa de peso por lo cual las intervenciones para la salud implementadas en este grupo demuestran que son eficaces.

Palabras clave: Obesidad, sobrepeso, educación en salud, prevención y control.

\section{Abstract}

Aim: To determine whether dietary guidance and physical activity education is an effective measure to reduce weight in overweight or obesity 18 to 29 years. Material and methods: cohort study; Two groups were formed: group A in which carried out an educational intervention and control group 
$B$; They were reassessed both groups over a period of 6 months. Results: A total of 776 (n) were divided into group A (389) and group B (387). In group A baseline weight $73.96 \mathrm{~kg} \mathrm{SD} \pm 17.28 \mathrm{BMI} 25.73 \mathrm{SD} \pm 4.8925 .73$. After the intervention found that weight was $71.59 \mathrm{SD} \pm 16.4$, fell $2.37 \mathrm{~kg} \mathrm{SD} \pm 1.73 \mathrm{Kg}$. Group B initial weight of 63.66 $\mathrm{SD} \pm 12.98$, and $\mathrm{BMI}$ of $22.81 \mathrm{SD} \pm 3.55$. In the second evaluation at 6 months an average weight of $64.24 \mathrm{SD} \pm 12.81$ was found, there was an average weight gain of 0.58 $\mathrm{SD} \pm 0.64$. $(p=0.000021)$. Conclusions: In group $A$, there was significant weight loss so the health interventions implemented in this group show that they are effective.

Keywords: Obesity, overweight, health education, prevention and controle.

\section{Resumo}

Objectivos: correlacionar se a educação na atividade física aconselhamento nutricional e é uma medida eficaz para a redução de peso entre os estudantes da Faculdade de Medicina da UANL de 18 a 29 anos matriculados no segundo ano da carreira do cirurgião e médico parteira. Material e métodos: estudo de coorte; I foi feito com todos os alunos UANL que completaram o segundo ano da carreira do cirurgião e médico parteira. Foram formados dois grupos: grupo A, em que realizou uma intervenção e controle de grupo educacional B; Eles foram reavaliados ambos os grupos após 6 meses para avaliar o seu progresso e conclusão do semestre. 6 meses. Resultados: Um total de 776 (n) foram divididos em Grupo A (389) e grupo B (387). No grupo A peso basal 73,96 kg D.E. IMC 17,28 \pm 25,73 e D.E. 4.89. Após a intervenção verificou-se que o peso foi de 71,59 D.E. $\pm 16,4$, uma queda de $2,37 \mathrm{~kg} \pm 1,73 \mathrm{~kg}$. Do grupo B peso inicial de 63,66 \pm 12,98, e IMC 22,81 \pm 3,55 DE. Na segunda avaliação do peso médio de 12,81 $\pm 64,24 \mathrm{DE}$ foi encontrado, houve um ganho de peso de 0,58 $\pm 0,64 \mathrm{~kg}$. Houve perda de peso no grupo A comparado ao grupo $B(p=0,000021)$. Conclusões: No grupo A, houve significativa perda de peso para as intervenções de saúde implementadas neste grupo mostram que eles são eficazes.

Palavras-chave: Obesidade, sobrepeso, educação em saúde, prevenção e controle.

\section{Introducción}

La obesidad y el sobrepeso se han convertido en los trastornos nutricionales más comunes en el mundo, según datos de la Organización Mundial de la Salud (OMS) el $65 \%$ de la población mundial vive en países donde el sobrepeso y la obesidad matan a más personas que la desnutrición (1), por lo que es considerada una epidemia mundial y un grave problema de salud pública tanto en países desarrollados como en vías de desarrollo (1).

Según la OMS la obesidad y el sobrepeso son el 5 to factor de riesgo relacionado a muertes en el mundo, al menos 2.8 millones de personas mueren cada año como resultado de tener sobrepeso u obesidad, además $44 \%$ de las personas diagnosticadas con diabetes mellitus, $23 \%$ de las enfermedades cardíacas isquémicas y entre el $7 \%$ al $41 \%$ de ciertos cánceres son atribuidos al sobrepeso y obesidad (2).

En Latinoamérica la prevalencia es alta igualándose a los países desarrollados siendo Paraguay el de más alto índice con un 68\% de su población, en segundo lugar, México con un $60 \%$ y Paraguay, en tercero con 53\%, con tendencia a aumentar en los siguientes años (3).

En México, la Encuesta Nacional de Salud y Nutrición (ENSANUT 2012) indicó que el sobrepeso y obesidad son problemas que afectan a cerca de $70 \%$ de la población 
(mujeres, 71,9\%; hombres, 66,7\%) entre los 30 y 60 años, en ambos sexos, siendo la obesidad la que afecta en mayor porcentaje al sexo femenino, en adolescentes esta tasa es de un 32\% dentro de este porcentaje se observó que en masculinos hay prevalencia de $21.2 \%$ sobrepeso y $10.0 \%$ en obesidad y en mujeres una prevalencia de $23.3 \%$ sobrepeso y $9.2 \%$ en obesidad (4).

En 2012, de acuerdo con los resultados de la ENSANUT, uno de cada tres hombres o mujeres adolescentes tiene sobrepeso u obesidad. Esto representa alrededor de 5981400 adolescentes en el país la proporción nacional de sobrepeso en adolescentes de 12 a 19 años, muestra que la edad más afectada tanto en hombres como en mujeres es la de 13 años. En relación a la obesidad las edades más afectadas en hombres y mujeres son las de 14 y 15 años respectivamente ().

En el estudio de Patton realizado en Victoria Australia, aproximada de 1,520 adolescentes seguidos desde los 14 años de edad por un período de 10 años adolescentes sin ningún tipo de intervención la prevalencia de sobrepeso durante la adolescencia fue de un $20 \%$ en ambos sexos y de obesidad en ambos sexos fue de un $4 \%$, dando como conclusión que 1 de cada 3 participantes en el estudio tuvo o tiene sobrepeso u obesidad durante alguna etapa del estudio y 1 de cada 5 participantes manifestaron obesidad o sobrepeso durante todas las etapas. Para la edad de 20 años o más estás tasas de obesidad y sobrepeso incrementaron, particularmente en hombres, a los 24 años las tasas de prevalencia de un 33\% en sobrepeso comparado con el $20 \%$ de la adolescencia y un $7 \%$ en obesidad comparado con el $4 \%$ de la adolescencia (6).

Se ha demostrado que intervenciones teórico-prácticas en escuelas ayudan al incremento de actividad física y mejoran los hábitos dietéticos de sus participantes. En conjunto las escuelas pueden implementar cambios en el ambiente en el que se desarrollan como disponibilidad de alimentos nutritivos que cumplan con los requerimientos calóricos de su edad y sexo, educación física, actividades extracurriculares y la aceptabilidad de hábitos saludables (7).

En nuestro país se realizó un estudio de intervención realizado en el estado de México, por los autores Shaman y cols para determinar la efectividad de estrategias aplicadas a la promoción de estilos de vida saludable en escuelas localizadas en el Estado de México, el estudio no fue concluyente pero tuvo un pequeño pero estadísticamente significante efecto en la probabilidad de pasar de sobrepeso a obesidad, también documentó una pequeña baja en el porcentaje de sobrepeso y mantener el índice de masa corporal normal (ㅇ).

Hay programa educativos como "Choose Health" que consiste en la intervención educativa en cambios conductuales en el estilo de vida enfocándose en los hábitos alimenticios y aumentar la actividad física por medio de ejercicios que mejoran la actividad cardiovascular, en el 2012 Brenan y cols seleccionaron 29 hombres y 34 mujeres con sobrepeso y obesidad entre las edades de 12 a 19 años en un período de 6 meses se vio que las personas habían reducido significativamente su IMC y mejora en la calidad de vida; otro estudio realizado por Peralta y cols fue medir la factibilidad, aceptabilidad y potencial eficacia de un programa contra la obesidad infantil, se realizó durante 6 meses con 176 participantes masculinos de entre 13-14 años. Se dividieron en dos grupos 1 control y el otro donde se aplicaron las estrategias, comparando los resultados se vio que en el grupo donde se aplicaron las estrategias había reducido su IMC mientras que en el control se documentó un aumento de IMC (9-12). 
La intervención educativa debe incluir una terapia motivacional y de afrontamiento para tener una mejor resultado además de la educación, en el estudio de Walkley y Fraser sobre terapia cognitivo-conductual en el tratamiento del sobrepeso y obesidad en adolescentes se aplicaron estrategias para lograr un apego a las estrategias de reducción de peso mediante entrevistas y pláticas motivacionales y terapia cognitiva, con una muestra de 63 participantes adolescentes hombres con sobrepeso, se dividieron en dos grupos uno al que se le aplicaron las entrevistas y terapia y el otro fue control Se demostró que en el grupo con ayuda bajo significativamente más de peso que los que se aplicó está medida ya que pudieron identificar sus puntos débiles les dieron ayuda y motivación para poder terminar sus rutinas de ejercicios (13-16).

A pesar de los esfuerzos que se han realizado en campañas de Salud Pública para prevenir la obesidad en escolares, varios estudios realizados con intervenciones en el estilo de vida usando diferentes métodos han reportado tener poco efecto reduciendo la obesidad y sobrepeso, solamente mostrando que los efectos de estas estrategias sirven para mantener el IMC uniforme. Aunque la mayoría de los estudios basados en intervenciones escolares a corto plazo han tenido efectos en el sobrepeso y obesidad en los adolescentes además de los niveles de glucosa, insulina o ambos, a largo plazo no han demostrado ser efectivos (17).

\section{Justificación}

Es importante conocer y realizar acciones de salud que estén a nuestro alcance para reducir la epidemia de la obesidad, por lo cual se realiza este estudio, y se podrá observar que ciertas acciones de salud pueden beneficiar a la población elegida.

\section{Objetivo general}

Correlacionar si la educación en orientación alimentaria y actividad física es una medida eficaz para la reducción de peso en estudiantes de 18 a 29 años de la facultad de Medicina de la UANL que cursan el segundo año de la carrera de médico cirujano y partero.

\section{Materiales y métodos}

Estudio de cohorte en el cual se evaluaron medidas antropométricas, y se clasificaron de acuerdo a su índice de masa corporal según la Organización Mundial de la Salud, en sujetos de la facultad de Medicina UANL y se dividieron en grupos A (intervención) y $B$ (control) de manera aleatorizada al momento que acudían a la toma de peso y talla; Se aplicaron a los participantes encuestas sobre sus hábitos físicos y alimenticios qué realizan rutinariamente durante una semana.

En el grupo de intervención se realizaron medidas caracterizadas por sesiones quincenales sobre temas de ámbito médico, nutricional y se realizó un seguimiento a los 6 meses para evaluar el impacto en pérdida de peso y/o disminución en IMC.

Las características de la estrategia en nutrición fueron:

- Gradual reducción del contenido energético de las comidas fuertes en el día

- Gradual adherencia a realizar un programa de actividad física

- Implementación del programa "mi estilo de vida: mi reto" para comer sanamente mis 3 comidas y 2 colaciones (Un vegetal, una fruta y tomar agua) y actividad física durante la semana. 


\section{Descripción de la intervención}

- Educación: Información acerca del sobrepeso y obesidad, definición, causas y consecuencias explicación del protocolo, objetivos y estrategias para alcanzar las metas de salida.

- Conducta alimentaria: Estrategias para cambiar los hábitos alimenticios, auto monitorizar la ingesta de alimentos y estrategias de control externo para manejar las señales de comer, conductas y consecuencias.

- Actividad física: Estrategias conductuales para reducir el tiempo sedentario.

- Selección de alimentos saludables.

- Estrategias de afrontamiento de un diagnostico crónico: reconocer pensamientos y emociones e identificar y usar estrategias positivas.

- Fueron divididas en 8 sesiones que incluyeron actividades de participación recreacional para que los participantes.

\section{Población y Muestra}

Se realizó un muestreo por conveniencia no probabilístico de estudiantes entre 18 y 29 años de la facultad de Medicina UANL. Se tomó el total población de alumnos que cursan en el segundo año de la carrera de médico cirujano y partero de la facultad de medicina que acudieron a la cita el día asignado y se dividieron en dos grupos de manera en grupo A (intervención) y grupo B (control).

\section{Criterios de inclusión}

Estudiantes con edad de 18 a 29 años

Estudiantes de la facultad de medicina UANL

\section{Criterios de Exclusión}

No aceptar participar en el estudio

Contar con menos de 18 años y/o mayor de 30 años.

Presencia de cualquier comorbilidad o discapacidad.

\section{Criterio de eliminación}

Individuos que acudan al estudio y se descubra durante el mismo que no cumple la edad mínima requerida.

\section{Técnica de análisis de datos}

Se utilizó la estadística descriptiva de la muestra con media y desviación estándar. Y se utilizaron gráficas y tablas para presentación de resultados. Se calculó prueba T de student para comparar la pérdida de peso en grupo A y grupo B después de la intervención.

\section{Consideraciones éticas}

En este protocolo no hubo conflicto de intereses. Se utilizó consentimiento informado para todos los sujetos y la información obtenida fue exclusivamente utilizada para el estudio sin afectar en alguna manera al paciente. Todo fue sometido al comité de ética de la Facultad de Medicina de la Universidad Autónoma de Nuevo León y aceptado. 


\section{Resultados}

Se obtuvo una población total de 776 sujetos (n), de los 356 fueron del género femenino $(45,6 \%)$ y 420 del género masculino $(53,4 \%$ ) (figura 1 ). La edad en promedio para ambos grupos fue de 19,3 años con una desviación estándar DE $\pm 1,24$, el peso inicial fue de $68,7 \mathrm{~kg}$ con una $\mathrm{DE} \pm 16,01$ e índice de masa corporal fue de 24.2 DE \pm 4,52.

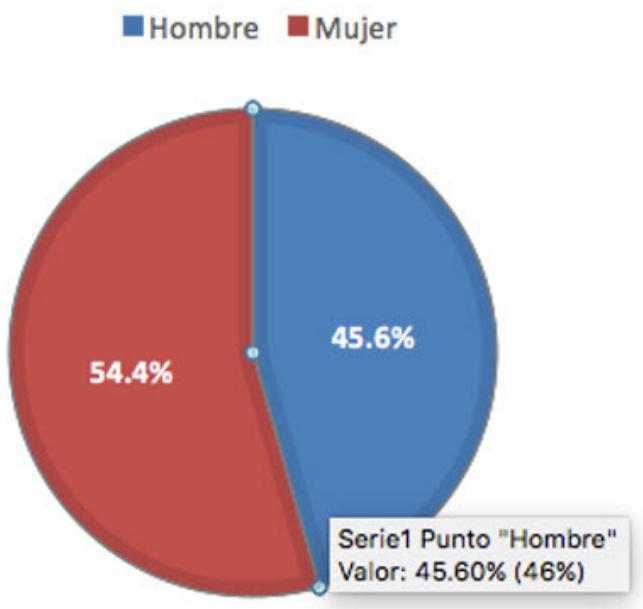

Figura 1. Porcentaje de individuos de género masculino y femenino

Se separaron los grupos de manera aleatoria (Tabla 1):

Tabla 1. Distribución de grupos de acuerdo a IMC

\begin{tabular}{|c|c|c|c|c|c|c|c|c|}
\hline \multirow{3}{*}{$\begin{array}{l}n=776 \\
\text { Mujeres }\end{array}$} & \multicolumn{4}{|c|}{ Grupo A } & \multicolumn{4}{|c|}{ Grupo B } \\
\hline & \multicolumn{4}{|c|}{$\%$} & & & \multicolumn{2}{|c|}{$\%$} \\
\hline & \multicolumn{2}{|c|}{155} & \multicolumn{2}{|c|}{40,2} & \multicolumn{2}{|c|}{198} & \multicolumn{2}{|c|}{51,2} \\
\hline Hombres & \multicolumn{2}{|c|}{231} & \multicolumn{2}{|c|}{59,8} & \multicolumn{2}{|c|}{189} & \multicolumn{2}{|c|}{48,8} \\
\hline \multirow[t]{2}{*}{ Peso } & \multicolumn{2}{|c|}{$74 \mathrm{Kg}$} & \multicolumn{2}{|c|}{$71,59 \mathrm{Kg}$} & \multicolumn{2}{|c|}{$63,5 \mathrm{Kg}$} & \multicolumn{2}{|c|}{$63,4 \mathrm{Kg}$} \\
\hline & Basal & $\%$ & 6 meses & $\%$ & Basal & $\%$ & 6 meses & $\%$ \\
\hline Bajo peso & 13 & 3,37 & 21 & 5,44 & 34 & 8,79 & 33 & 8,53 \\
\hline Normopeso & 173 & 44,82 & 196 & 50,78 & 262 & 67,70 & 267 & 68,99 \\
\hline Sobrepeso & 122 & 31,61 & 108 & 27,98 & 77 & 19,90 & 72 & 18,60 \\
\hline Obesidad 1 & 63 & 16,32 & 53 & 13,73 & 13 & 3,36 & 14 & 3,62 \\
\hline Obesidad 2 & 13 & 3,37 & 7 & 1,81 & 1 & 0,26 & 1 & 0,26 \\
\hline Obesidad 3 & 2 & 0,52 & 1 & 0,26 & 0 & 0,00 & 0 & 0,00 \\
\hline
\end{tabular}

Grupo A al cual se le se realizaron medidas caracterizadas por sesiones quincenales sobre temas de ámbito médico, nutricional y se realizó un seguimiento a los 6 meses para evaluar el impacto en pérdida de peso y/o disminución en IMC. El total de sujetos para este grupo fue de 386, de los cuales 155 (40,2\%) fueron hombres y 231 $(59,8 \%)$ fueron mujeres. La edad media fue 19,29 años DE \pm de 1,32, el peso corporal inicial fue en promedio $73,96 \mathrm{Kg} \mathrm{DE} \pm 17,28$ y su índice de masa corporal fue de 25,73 DE 4,89. Posterior a la intervención y evaluación a los 6 meses se encontró que 
el peso fue de $71,59 \mathrm{Kg} \mathrm{DE} \pm 16,4$, con una disminución de peso en promedio de 2,37 $\mathrm{Kg} \mathrm{DE} \pm 1,73 \mathrm{Kg}$ de acuerdo a la evaluación inicial; en cuanto a su índice de masa corporal se encontró en 24,91 DE \pm 4,63 (figura $\underline{2}$ y $\underline{3}$ ).

En el Grupo B (control) en el cual no se realizaron medidas de intervención fue un total de 387 sujetos de los cuales $198(51,2 \%)$ fueron mujeres y $189(48,8 \%)$ fueron hombres. En cuanto a su edad el promedio fue 19,2 años DE $\pm 1,16$, peso inicial 63,66 $\mathrm{Kg} \mathrm{DE} \pm 12,98$, e IMC 22,81 DE $\pm 3,55$. En la segunda evaluación a los 6 meses se encontró un peso promedio de 64,24 DE $\pm 12,81$, hubo aumento de peso en promedio de $0,58 \mathrm{Kg} \mathrm{DE} \pm 0,64$, y de IMC 23,1 DE $\pm 3,51$ (figura $2 \underline{\text { y }} \underline{3}$ ).

Como resultado tenemos que hubo reducción de peso en el grupo A en comparación con el grupo B con una $p=0,000021$.

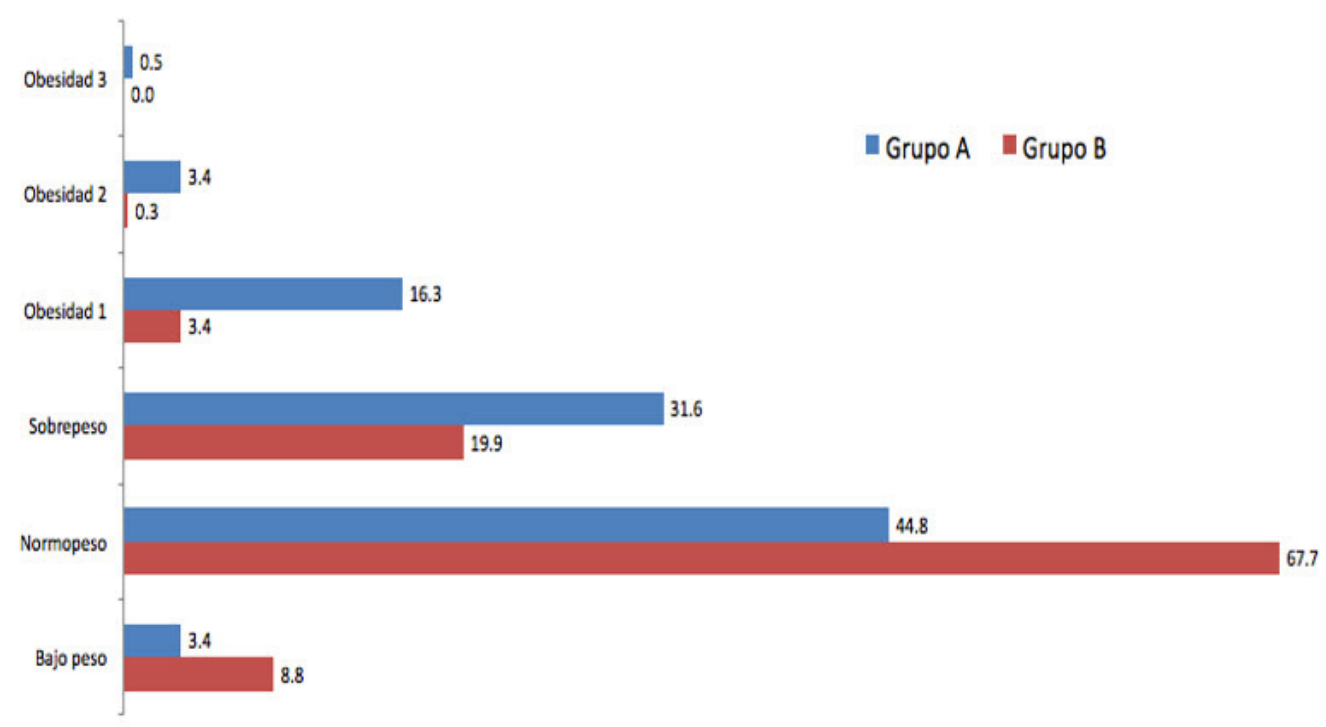

Figura 2. Distribución en porcentajes del grupo A y B en caslificación de IMC previo a intervención en salud

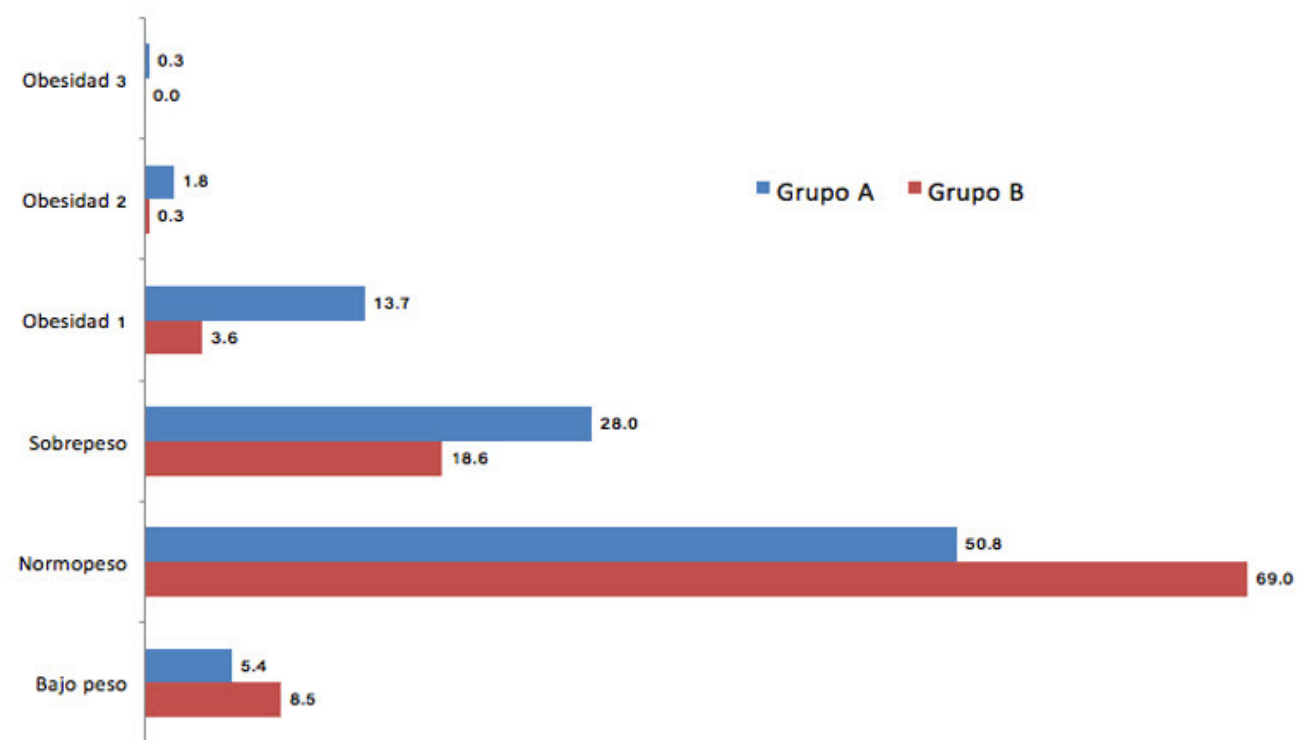

Figura 3. Distribución en porcentaje del grupo A y grupo B en clasificación de IMC posterior a intervención en salud. 
En el grupo A la mayoría tuvo cambios en su peso de manera significativa hubo cambio en 54 personas que tenía problemas de sobrepeso y obesidad y la pérdida de peso más significativa fue de 11,2 Kg en un individuo; el promedio de descenso de peso fue de $2,37 \mathrm{~kg}$

Los participantes del grupo B solo 7 bajaron de peso, el resto no tuvo cambios significativos; La pérdida de peso más significativa fue de 1,7 Kg aquí y se encontró en un individuo un aumento de $5 \mathrm{~kg}$; el promedio de descenso de peso fue de $0,09 \mathrm{~kg}$ y el promedio de aumento de peso: 0,95kg

Encontramos que el grupo A en general hubo pérdida de 11,2 kg en comparativo con el grupo B que no participo en las actividades donde solo hubo un cambio de $1 \mathrm{~kg}$ pero se encontró que este grupo hubo un aumento significativo de peso, donde de 98 participantes aumentaron de peso; esto da la finalidad que una intervención en tres aspectos educacional, emocional y físico tiene mejores resultados que no hacerlas

Se realizó una prueba de hipótesis que se escogió la de kolmogorov smirnov para comparar el índice de masa corporal basal y el índice de masa corporal después de la intervención educativa a los 6 meses encontrándose una $p<0,05$ siendo estadísticamente significativa que una intervención educativa planeada en base a principios de nutrición y actividad física es una medida efectiva para la reducción del estado nutricional.

\section{Discusión}

Hay programas educativos como "Choose Health" que consiste en la intervención educativa en cambios conductuales en el estilo de vida enfocándose en los hábitos alimenticios donde Se formaron dos grupos aleatoriamente uno de tratamiento y otro de contro; En un período de 6 meses establecido se vio que las personas habían reducido significativamente su IMC y mejora en la calidad de vida.

En nuestro estudio se observó una reducción de peso al combinar una terapia de orientación nutricional y psicológica de afrontamiento y actividad física 2 veces por semana; los autores Brenan y Peralta coinciden que si se realiza una intervención con un enfoque educativo en nutrición se ve favorecido la población con un descenso del IMC.

En el estudio de Walkley y Fraser sobre terapia cognitivo-conductual en el tratamiento del sobrepeso y obesidad en adolescentes se aplicaron estrategias para lograr un apego a las estrategias de reducción de peso mediante entrevistas y pláticas motivacionales y terapia cognitiva, con una muestra de 63 participantes adolescentes hombres con sobrepeso, se dividieron en dos grupos uno al que se le aplicaron las entrevistas y terapia y el otro fue control. Se demostró que en el grupo con ayuda bajo significativamente más de peso en el presente estudio que realizamos se hizo una conferencias magistrales y seguimiento por sicología en las personas del grupo A observándose una mayor disminución en la percepción y el estado de poder bajar de peso para mejorar su calidad de vida; esto nos da la pauta que si se interviene en el aspecto nutricional y psicológico se obtendrá mayor impacto en la reducción de peso.

\section{Conclusiones}

En el grupo A hubo perdida significativa de peso por lo cual las intervenciones para la salud implementadas en este grupo demuestran que son eficaces. 


\section{Bibliografía}

1. Importancia de la diabetes Mellitus en la salud pública. Dras. Szurpik juana, hebat adriana y col. Sección nutrición.

2. Cuentas en diabetes mellitus, enfermedades cardiovasculares y obesidad. Mexico2006 instituto de salud pública.

3. Panorámico epidemiológico y estadístico de la mortalidad. México 2010.

4. Organización mundial de la salud. Programa de diabetes Mellitus. Who.int

5. Norma Oficial Mexicana nom-0-ssa2-1994, para la prevención, tratamiento y control de la obesidad.

6. Asociación americana de diabetes (American Diabetes Asociación). Documento oficial 2012 .www.diabetes.org

7. Programa integrados de salud. Guía técnica del Imss. Dra Aceves; Dr Celis Quintal. IMSS 2002.

8. La salud pública y el trabajo comunitario. Rafael González, Laura Moreno. Capitulo historia natural de la enfermedad de diabetes Mellitus. Mc Graw Hill 2010.

9. Diabetes Mellitus. Guía para el manejo del paciente. Nancy Holmes, Joan Robinson, Beverly Tscheschlog. Capitulo 1. Editorial Wolters klumer 2009.

10. Diabetes Mellitus. Guía para el manejo integral del paciente. Melchor Alpizar Salazar. Editorial Alfill. Segunda edición 2007. https://www.researchgate.net/ publication/44564886 Guia para el manejo integral del paciente diabetico Melchor Alpizar Salazar

11. American diabetes association. " report of the expert committe on the diagnosis and classification of diabetes Mellitus" Diabetes Care 26 (suppl 1 ): s5-s20. 2012 https://www.ncbi.nlm.nih.gov/pubmed/12502614

12. Beck NH: metodologies in characterizacion and clasification of prediabetic states to noninsul-dependent diabetes Mellitus (NIDDM). Diabetes forum series research 2004.5:1476-169.

13. Alpizar SM, Sotomayor Ga. Diabetes mellitus, prioridad institucional. Rev salud comunitaria IMSS 2008; 2(1):31-35Patton GC, Coffey C, Carlin JB, Sawyer SM,

14. Williams J, Olsson CA, Wake M. "Overweight and obesity between adolescence and young adulthood: a 10-year prospective cohort study." J Adolesc Health. 2011 Mar;48(3):275-80. https://www.ncbi.nlm.nih.gov/pubmed/21338899

15. The NS, Suchindran C, North KE, Popkin BM, Gordon-Larsen P. "Association of adolescent obesity with risk of severe obesity in adulthood." JAMA. 2010 Nov 10;304(18):2042-7. http://jamanetwork.com/journals/jama/fullarticle/186874 
16. Shamah Levy T, Morales Ruán C, Amaya Castellanos C, Salazar Coronel A, Jiménez Aguilar A, Méndez Gómez Humarán I. "Effectiveness of a diet and physical activity promotion strategy on the prevention of obesity in Mexican schoolchildren." BMC Public Health. 2012 Mar 1;12:152. http://bmcpublichealth.biomedcentral.com/articles/10.1186/1471-2458-12-152

17. Kimm SY, Glynn NW, Obarzanek E, Kriska AM, Daniels SR, Barton BA, Liu K. "Relation between the changes in physical activity and body-mass index during adolescence: a multicentre longitudinal study." Lancet. 2005 Jul 23-29;366(9482):3017. https://www.ncbi.nlm.nih.gov/pubmed/16039332

18. Brennan L, et al. "Physiological and behavioural outcomes of a randomized controlled trial of a cognitive behavioural lifestyle intervention for overweight and obese adolescents." Obes ResClin Pract (2012). http://www.sciencedirect.com/ science/article/pii/S1871403X12000191

19. Freedman DS, Mei Z, Srinivasan SR, Berenson GS, Dietz WH. "Cardiovascular risk factors and excess adiposity among overweight children and adolescents: the Bogalusa Heart Study." J Pediatr. 2007 Jan;150(1):12-17.e2. https://www.ncbi. nlm.nih.gov/pubmed/17188605

20. Brennan L, Walkley J, Fraser SF, Greenway K, Wilks R. "Motivational interviewing and cognitive behaviour therapy in the treatment of adolescent overweight and obesity: study design and methodology." Contemp Clin Trials. 2008 May;29(3):359https://www.ncbi.nlm.nih.gov/pubmed/17950046

21. Andrade FC, Vazquez-Vidal I, Flood T, Aradillas-Garcia C, Vargas-Morales JM, Medina-Cerda E, Teran-Garcia M. "One-year follow-up changes in weight are associated with changes in blood pressure in young Mexican adults." Public Health. 2012 Jun;126(6):535-40 https://www.ncbi.nlm.nih.gov/pubmed/22546507

22. Adams MA, Caparosa S, Thompson S, Norman GJ. "Translating physical activity recommendations for overweight adolescents to steps per day." Am J Prev Med. 2009 Aug;37(2):137-40. https://www.ncbi.nlm.nih.gov/pubmed/19524391 